\title{
EIGENFUNCTION AND BOCHNER RIESZ ESTIMATES ON MANIFOLDS WITH BOUNDARY
}

\author{
Christopher D. Sogge
}

\section{Introduction}

The purpose of this paper is to give a simple proof of sharp $L^{\infty}$ estimates for the eigenfunctions of the Dirichlet Laplacian on smooth compact Riemannian manifolds $(M, g)$ of dimension $n \geq 2$ with boundary $\partial M$ and then to use these estimates to prove new estimates for Bochner Riesz means in this setting. Thus, we shall consider the Dirichlet eigenvalue problem

$$
\left(\Delta+\lambda^{2}\right) u(x)=0, x \in M, \quad u(x)=0, x \in \partial M,
$$

with $\Delta=\Delta_{g}$ being the Laplace-Beltrami operator associated to the Riemannian metric $g$. Recall that the spectrum of $-\Delta$ is discrete and tends to infinity. Let $0<\lambda_{1}^{2} \leq \lambda_{2}^{2} \leq \lambda_{3}^{2} \leq \ldots$ denote the eigenvalues, so that $\left\{\lambda_{j}\right\}$ is the spectrum of the first order operator $\mathcal{P}=\sqrt{-\Delta}$. Let $\left\{e_{j}(x)\right\}$ be an associated real orthonormal basis, and let

$$
e_{j}(f)(x)=e_{j}(x) \int_{M} f(y) e_{j}(y) d y,
$$

be the projection onto the $j$-th eigenspace. Here and in what follows $d y$ denotes the volume element associated with the metric $g$.

Grieser [5] recently proved the $L^{\infty}$ estimates

$$
\left\|e_{j}(f)\right\|_{\infty} \leq C \lambda_{j}^{(n-1) / 2}\|f\|_{2}
$$

which are sharp for instance when $M$ is the upper hemisphere of $S^{n}$ with the standard metric. One of our main results is a slight strengthening of this. We shall consider the unit band spectral projection operators,

$$
\chi_{\lambda} f=\sum_{\left|\lambda_{j}-\lambda\right| \leq 1} e_{j}(f),
$$

and show that these enjoy the same bounds:

Theorem 1.1. Fix a compact Riemannian manifold $(M, g)$ with boundary of dimension $n \geq 2$. Then there is a uniform constant $C$ so that

$$
\left\|\chi_{\lambda} f\right\|_{\infty} \leq C \lambda^{(n-1) / 2}\|f\|_{2}, \quad \lambda \geq 1 .
$$

Received February 21, 2002.

The author was supported in part by the NSF. 
In the case of manifolds without boundary, this and more general estimates of the form

$$
\left\|\chi_{\lambda} f\right\|_{p} \leq C \lambda^{\sigma(p)}\|f\|_{2}, \quad \lambda \geq 1, p \geq 2,
$$

where

$$
\sigma(p)=\max \left\{\frac{n-1}{2}-\frac{n}{p}, \frac{n-1}{2}\left(\frac{1}{2}-\frac{1}{p}\right)\right\}
$$

were proved in [15]. These estimates cannot be improved since one can show that the operator norms satisfy $\lim \sup _{\lambda \rightarrow \infty} \lambda^{-\sigma(p)}\left\|\chi_{\lambda}\right\|_{L^{2} \rightarrow L^{p}}>0$ (see [17]).

The special case of (1.5) where $p=\infty$ seems to have been first stated in [15], but it can be proved using much older estimates of Avakumovic [1], [2] and Levitan [11] that arise in the proof of the sharp Weyl formula for Riemannian manifolds without boundary. After that, Hörmander [7] proved the sharp Weyl formula for general self-adjoint elliptic operators on manifolds without boundary. Recently, in the case of manifolds without boundary, the author and Zelditch [18] proved estimates that imply that for generic metrics on any manifold one has the bounds $\left\|e_{j}\right\|_{\infty}=o\left(\lambda_{j}^{(n-1) / 2}\right)$ for $L^{2}$-normalized eigenfunctions. The corresponding result for manifolds with boundary is not known.

In the case of manifolds with boundary, the only known results for the unit band spectral projection operators were due to D. Grieser [4] and H. Smith and the author [14], who showed that the bounds (1.5) hold under the assumption that the manifold has geodesically concave boundary. The two-dimensional case was handled in [4], and the higher dimensional in [14].

In the other direction, Grieser in his thesis [4], showed that (1.5) cannot hold if the boundary of $M$ has a point that is geodesically convex. In this case, for instance, when $n=2$, he constructed a counterexample showing that the bounds in in (1.5) can only hold for $p \geq 8$. Showing that they are valid for this range of exponents appears to be very difficult. The reason for the difference in the smaller range of exponents for this case is related to the existence of Rayleigh whispering galleries. Specifically, one can construct functions with spectrum in $\lambda$-unit bands that are concentrated in a $\lambda^{-2 / 3}$ neighborhood of the boundary, while in the boundaryless case, the counterexample showing that (1.5) is sharp involves showing that there are functions of this type concentrating in $\lambda^{-1 / 2}$ neighborhoods of geodesics.

We shall follow an idea of Grieser [5] to prove our generalization (1.4) of his result (1.2). Grieser first showed that one always has the uniform bounds $\left|e_{j}(x)\right| \leq C \lambda_{j}^{(n-1) / 2}$ when the distance from $x$ to $\partial M$ is bounded from below by $\lambda_{j}^{-1}$. This first step was achieved by adapting the proof of estimates for the local Weyl law which are due to Seeley [13], Pham The Lai [12] and Hörmander [8]. He then used these bounds and a form of the maximum principle ([6], Theorem 10, p. 73) for solutions of (1.1) to obtain the bounds in the $\lambda_{j}^{-1}$ neighborhood of the boundary. 
Our proof of (1.4) will be to first see that the aforementioned local Weyl estimate of Seeley, Pham The Lai, and Hörmander immediately gives the stronger estimate

$$
\left|\chi_{\lambda} f(x)\right| \leq C \lambda^{(n-1) / 2}\|f\|_{2}, \quad \lambda \geq 1
$$

for all $x$ when $n=2$ or when for $n \geq 3$ the distance to the boundary is bounded below by $\lambda^{-(n-1) /(n-2)}$. If $n=3$, one can use this fact and a simple argument involving Sobolev's theorem (cf. Theorem 17.5.3 in [8]) to show that (1.7) must also hold in the missing piece where $\operatorname{dist}(x, \partial M) \leq \lambda^{-2}$. For $n \geq 4$; however, one must use a maximum principle argument as introduced by Greiser for these problems. We shall not directly use the form of the maximum principle employed by Grieser, but rather see that its proof can be used to show that the uniform bounds (1.7) must also hold in a $\lambda^{-1}$ neighborhood of the boundary, which would finish the proof of (1.4).

Our other main result will be some new estimates for Bochner Riesz means of eigenfunctions. Recall the Bochner Riesz means of index $\delta \geq 0$ are defined by

$$
S_{\lambda}^{\delta} f=\sum_{\lambda_{j} \leq \lambda}\left(1-\frac{\lambda_{j}^{2}}{\lambda^{2}}\right)^{\delta} e_{j}(f) .
$$

It is known that a necessary condition for these operators to be uniformly bounded on $L^{p}$ for a given $1 \leq p \leq \infty, p \neq 2$, is that

$$
\delta(p)=\max \{n|1 / 2-1 / p|-1 / 2,0\} .
$$

Note that when $p \geq 2(n+1) /(n-1)$, one has $\delta(p)=\sigma(p)$, where $\sigma(p)$ is the exponent in (1.6). Using this fact, the author used the boundarlyless estimates (1.5) to prove the first sharp estimates for Bochner Riesz means on compact Riemannian manifolds in [16]. Specifically, it was shown that for a given $p \in$ $[1,2(n+1) /(n+3)] \cup[2(n+1) /(n-1), \infty]$ one has the uniform bounds

$$
\left\|S_{\lambda}^{\delta} f\right\|_{p} \leq C\|f\|_{p}
$$

in this setting, provided that $\delta>\delta(p)$. Earlier, weaker results were due to many people, including Hörmander [9].

Since we only know that the desired bounds for eigenfunctions (1.5) hold for $p=\infty$, we can only at this stage prove the sharp estimates for Bochner Riesz means when $p=1$ or $p=\infty$ :

Theorem 1.2. Fix a smooth compact Riemannian manifold with boundary $(M, g)$ of dimension $n \geq 2$. Then if $\delta>(n-1) / 2$ one has the uniform bounds

$$
\left\|S_{\lambda}^{\delta} f\right\|_{p} \leq C\|f\|_{p}
$$

for every $1 \leq p \leq \infty$.

By interpolating with the trivial estimate for $p=2$, and using duality one gets the bounds (1.10) from the special case where $p=1$. However, the bounds for $1<p<\infty$ certainly are not sharp. 
We shall adapt the argument from [16] to show that (1.5) implies (1.10). In [16] the Tauberian arguments behind the proof of the sharp Weyl formula were adapted to show that one could write $S_{\lambda}^{\delta}=\tilde{S}_{\lambda}^{\delta}+R_{\lambda}^{\delta}$, where the "remainder" term $R_{\lambda}^{\delta}$ could be controlled by (1.5), while the other piece, $\tilde{S}_{\lambda}^{\delta}$, could be estimated by computing its kernel explicitly via the Hadamard parametrix and then estimating the resulting integral operator using straightforward adaptations of the arguments for the Euclidean case. In the setting of manifolds with boundary, this approach does not seem to work since the known parametrices for the wave equation do not seem strong enough unless one assumes that the boundary is geodesically concave. Here, we shall get around this fact by simplifying the earlier arguments and show that estimates for Bochner Riesz operators just follow from (1.5) and the finite propagation speed of solutions of the Dirichlet wave equation.

In what follows we shall use the convention that $C$ will denote a constant that is not necessarily the same at each occurrence.

It is a pleasure to thank S. Zelditch for a number helpful conversations. I am also grateful to $\mathrm{M}$. Taylor and $\mathrm{X}$. Xu for helpful comments regarding an early draft of this paper.

\section{2. $L^{\infty}$ estimates for unit band spectral projection operators}

In this section we shall prove Theorem 1.1. Thus, we need to see that one has the uniform bounds

$$
\left|\chi_{\lambda} f(x)\right| \leq C \lambda^{(n-1) / 2}\|f\|_{2}, \quad \lambda \geq 1
$$

Note that

$$
\chi_{\lambda} f(x)=\int_{M} \sum_{\left|\lambda_{j}-\lambda\right| \leq 1} e_{j}(x) e_{j}(y) f(y) d y,
$$

therefore, by the converse to Schwarz's inequality and orthogonality, one has the bounds (2.11) at a given point $x$ if and only if

$$
\sum_{\left|\lambda-\lambda_{j}\right| \leq 1}\left(e_{j}(x)\right)^{2} \leq C^{2} \lambda^{n-1}
$$

Because of this, (2.11) would be a consequence of the following two results.

Proposition 2.3. Fix $(M, g)$ as above. Then, given $\varepsilon>0$, there is a uniform constant $C$ so that for $\lambda \geq 1$

$$
\sum_{\left|\lambda-\lambda_{j}\right| \leq 1}\left(e_{j}(x)\right)^{2} \leq C_{\varepsilon} \lambda^{n-1}
$$

for $x$ satisfying

$$
d(x) \geq \varepsilon \lambda^{-1}
$$

if $n \geq 3$ where $d(x)$ denotes the Riemannian distance to $\partial M$. 
Proposition 2.4. If $(M, g)$ is as above then for large $\lambda$ we have

$$
\max _{\left\{x: d(x) \leq \frac{1}{2}(\lambda+1)^{-1}\right\}} \sum_{\left|\lambda-\lambda_{j}\right| \leq 1}\left(e_{j}(x)\right)^{2} \leq 4 \max _{\left\{x: d(x)=\frac{1}{2}(\lambda+1)^{-1}\right\}} \sum_{\left|\lambda-\lambda_{j}\right| \leq 1}\left(e_{j}(x)\right)^{2} .
$$

Proof of Proposition 2.3. We shall see that (2.13) is an immediate consequence of Theorem 17.5.10 in Hörmander [8], which in turn is based on earlier work of Seeley [13] and Pham The Lai [12]. To state this result, we let

$$
e(x, \lambda)=(2 \pi)^{-n} \int_{\left\{\xi \in \mathbb{R}^{n}:|\xi| \leq \lambda\right\}}\left(1-e^{i 2 d(x) \xi_{n}}\right) d \xi .
$$

If we assume that local coordinates have been chosen so that the Riemannian volume form is $d x_{1} \ldots d x_{n}$, then the result just quoted says that there is a uniform constant $C$ so that for $\lambda \geq 1$

$$
\left|\sum_{\lambda_{j} \leq \lambda}\left(e_{j}(x)\right)^{2}-e(x, \lambda)\right| \leq C \lambda\left(\lambda+d(x)^{-1}\right)^{n-2} .
$$

Since $\lambda\left(\lambda+d(x)^{-1}\right)^{n-2}=O\left(\lambda^{n-1}\right)$ for all $x$ satisfying (2.14) this yields Proposition 2.3 since

$$
|e(x, \lambda+1)-e(x, \lambda-1)| \leq C \lambda^{n-1} .
$$

Although, we do not need to use it here, the proof of (2.17) actually gives the bounds (2.13) when $d(x) \geq \lambda^{-\frac{n-1}{n-2}}$ for $n \geq 3$ and for all $x$ when $n=2$. This stronger fact just follows from the estimate (17.5.20) in [8].

Proof of Proposition 2.4. It is convenient to use geodesic coordinates with respect to the boundary. Specifically, we shall use the fact that we can find a small constant $c>0$ so that the map $\left(x^{\prime}, x_{n}\right) \in \partial M \times[0, c) \rightarrow M$, sending $\left(x^{\prime}, x_{n}\right)$ to the endpoint, $x$, of the geodesic of length $x_{n}$ which starts at $x^{\prime} \in \partial M$ and is perpendicular to $\partial M$ is a local diffeomorphism. Note then that $d(x)=x_{n}$. Under this identification the metric takes the form

$$
\sum_{i, j=1}^{n} g_{i j}(x) d x^{i} d x^{j}=\left(d x^{n}\right)^{2}+\sum_{i, j=1}^{n-1} g_{i j}^{\prime}\left(x^{\prime}, x_{n}\right) d x^{i} d x^{j}
$$

where $g_{i j}^{\prime}\left(\cdot, x_{n}\right)$ is a Riemannian metric on $\partial M$ depending smoothly on $x_{n} \in$ $[0, c)$. Consequently, in this neighborhood of the boundary, the Laplacian can be written as

$$
\Delta=\sum_{i, j=1}^{n} g^{i j}(x) \frac{\partial^{2}}{\partial x_{i} \partial x_{j}}+\sum_{i=1}^{n} b_{i}(x) \frac{\partial}{\partial x_{i}},
$$

using local coordinates for $\partial M$, where $g^{i j}$ the matrix with entries $\left(g^{i j}\right)_{1 \leq i, j \leq n-1}=$ $\left(g_{i j}^{\prime}\right)^{-1}$ and $g^{n n}=1$, and $g^{n k}=g^{k n}=0, k \neq n$. Also the $b_{j}(x)$ are $C^{\infty}$ and real valued. 
In what follows we shall assume that $\lambda$ is large enough so that $\lambda \geq 2 / c$. Assume further that spec $\sqrt{-\Delta} \cap[\lambda-1, \lambda+1] \neq \emptyset$, and consider the function

$$
H(x)=\frac{1}{(w(x))^{2}} \sum_{\lambda_{j} \in[\lambda-1, \lambda+1]}\left(e_{j}(x)\right)^{2},
$$

where

$$
w(x)=1-(\lambda+1)^{2} x_{n}^{2} .
$$

Suppose that in the strip $\left\{x \in M: 0 \leq x_{n} \leq \frac{1}{2}(\lambda+1)^{-1}\right\}$ the function $H(x)$ has a maximum at an interior point $x=x_{0}$. Then

$$
v(x)=\frac{1}{w(x)} \sum_{\lambda_{j} \in[\lambda, \lambda+1]} \frac{e_{j}\left(x_{0}\right)}{w\left(x_{0}\right)} e_{j}(x)
$$

must have a positive maximum at $x=x_{0}$. For because of our assumptions on the spectrum we then have $v\left(x_{0}\right)=\sum_{\lambda_{j} \in[\lambda, \lambda+1]}\left(e_{j}\left(x_{0}\right) / w\left(x_{0}\right)\right)^{2}>0$, while at other points in the strip

$$
\begin{aligned}
&|v(x)| \leq \frac{1}{w(x)}\left(\sum_{\lambda_{j} \in[\lambda, \lambda+1]}\left(e_{j}(x)\right)^{2}\right)^{1 / 2} \frac{1}{w\left(x_{0}\right)}\left(\sum_{\lambda_{j} \in[\lambda, \lambda+1]}\left(e_{j}\left(x_{0}\right)\right)^{2}\right)^{1 / 2} \\
&=(H(x))^{1 / 2}\left(H\left(x_{0}\right)\right)^{1 / 2} \leq H\left(x_{0}\right)=v\left(x_{0}\right) .
\end{aligned}
$$

Note that in the $\operatorname{strip}\left\{x \in M: 0 \leq x_{n} \leq \frac{1}{2}(\lambda+1)^{-1}\right\}$ we have

$$
\begin{aligned}
\left(\Delta+\lambda_{j}^{2}\right) w=-2(\lambda+1)^{2}-2 b_{n}(x) x_{n}(\lambda+1)^{2} & +\lambda_{j}^{2}\left(1-(\lambda+1)^{2} x_{n}^{2}\right) \\
& \leq-(\lambda+1)^{2} / 2, \quad \lambda_{j} \leq \lambda+1,
\end{aligned}
$$

assuming that $\lambda$ is large enough so that $\left|2 b_{1}(x) x_{n}\right| \leq 1 / 2$. Also, in this strip we have that $\frac{1}{2} \leq w(x) \leq 1$.

Let us set

$$
v_{j}(x)=\frac{e_{j}(x)}{w(x)} \frac{e_{j}\left(x_{0}\right)}{w\left(x_{0}\right)},
$$

so that $v(x)=\sum_{\lambda_{j} \in[\lambda-1, \lambda+1]} v_{j}(x)$. We also set

$$
u_{j}(x)=\frac{e_{j}\left(x_{0}\right)}{w\left(x_{0}\right)} e_{j}(x),
$$

and note that $\left(\Delta+\lambda_{j}^{2}\right) u_{j}(x)=0$.

A computation (see p. $72,[6]$ ) shows that for a given $j$ we have

$$
\begin{aligned}
0= & \frac{1}{w}\left(\Delta+\lambda_{j}^{2}\right) u_{j} \\
& =\sum_{k, l=1}^{n} g^{k l}(x) \frac{\partial^{2} v_{j}}{\partial x_{k} \partial x_{l}}+\sum_{k=1}^{n}\left(\frac{2}{w} \sum_{l=1}^{n} g^{k l}(x) \frac{\partial w}{\partial x_{l}}+b_{k}\right) \frac{\partial v_{j}}{\partial x_{k}}+\frac{v_{j}}{w}\left(\Delta+\lambda_{j}^{2}\right) w .
\end{aligned}
$$


Therefore, if we sum over $\lambda_{j} \in[\lambda-1, \lambda+1]$, we get

$$
\sum_{k, l=1}^{n} g^{k l}(x) \frac{\partial^{2} v}{\partial x_{k} \partial x_{l}}+\sum_{k=1}^{n}\left(\frac{2}{w} \sum_{l=1}^{n} g^{k l}(x) \frac{\partial w}{\partial x_{l}}+b_{k}\right) \frac{\partial v}{\partial x_{k}}=-\sum_{\lambda_{j} \in[\lambda-1, \lambda+1]} \frac{v_{j}}{w}\left(\Delta+\lambda_{j}^{2}\right) w .
$$

In particular, at $x=x_{0}$, we have

$$
\begin{aligned}
\sum_{k, l=1}^{n} g^{k l}\left(x_{0}\right) \frac{\partial^{2} v\left(x_{0}\right)}{\partial x_{k} \partial x_{l}} & +\sum_{k=1}^{n}\left(\frac{2}{w} \sum_{l=1}^{n} g^{k l}\left(x_{0}\right) \frac{\partial w}{\partial x_{l}}+b_{k}\right) \frac{\partial v\left(x_{0}\right)}{\partial x_{k}} \\
& =\frac{-1}{w\left(x_{0}\right)} \sum_{\lambda_{j} \in[\lambda-1, \lambda+1]}\left(\frac{e_{j}\left(x_{0}\right)}{w\left(x_{0}\right)}\right)^{2}\left(\Delta+\lambda_{j}^{2}\right) w\left(x_{0}\right)>0 .
\end{aligned}
$$

But this is impossible since $v$ has a positive maximum at $x_{0}$, which implies that $\partial v\left(x_{0}\right) / \partial x_{k}=0$ for every $k$, and $\sum_{k, l=1}^{n} g^{k l}\left(x_{0}\right) \frac{\partial^{2} v\left(x_{0}\right)}{\partial x_{k} \partial x_{l}} \leq 0$. Thus, we conclude that the function $H(x)$ cannot have a maximum at an interior point of the strip, $\left\{x: 0 \leq x_{n} \leq \frac{1}{2}(\lambda+1)^{-1}\right\}$. Because of this, the Dirichlet conditions, and our lower bound for $w$, we get that

$$
\sup _{\left\{x: 0 \leq x_{n} \leq \frac{1}{2}(\lambda+1)^{-1}\right\}} \sum_{\lambda_{j} \in[\lambda-1, \lambda+1]}\left(e_{j}(x)\right)^{2} \leq 4 \sup _{\left\{x: x_{n}=\frac{1}{2}(\lambda+1)^{-1}\right\}} \sum_{\lambda_{j} \in[\lambda-1, \lambda+1]}\left(e_{j}(x)\right)^{2},
$$

as desired, which completes the proof of Proposition 2.4.

\section{Estimates for Bochner Riesz means}

In this section we shall see how favorable estimates for the unit band spectral projection operators imply sharp estimates for Bochner Riesz means. Specifically, we shall prove the following result which implies Theorem 1.2.

Proposition 3.5. Assume that for a given $1 \leq p<2$ one has the uniform bounds

$$
\left\|\chi_{\lambda} f\right\|_{2} \leq C \lambda^{\frac{n}{p}-\frac{n+1}{2}}\|f\|_{p}, \quad \lambda \geq 1 .
$$

Then for a given $\delta>\frac{n}{p}-\frac{n+1}{2}$ there is a uniform constant $C_{\delta}$ so that

$$
\left\|S_{\lambda}^{\delta} f\right\|_{p} \leq C_{\delta}\|f\|_{p} .
$$

This implies Theorem 1.2, since, by duality, (1.4) implies that (3.18) must hold when $p=1$. This implies that if $\delta>(n-1) / 2$ the $S_{\lambda}^{\delta}$ are uniformly bounded on $L^{1}$, which implies the same for all $L^{p}, 1 \leq p \leq \infty$ by duality and interpolation.

The proposition also shows that if (3.18) holds then one has the sharp estimate that the $S_{\lambda}^{\delta}$ operators are uniformly bounded on $L^{p}$ when $\delta>\delta(p)$, with $1 \leq$ $p \leq 2 n /(n+1)$, if, as in (1.9), $\delta(p)$ is the so-called critical index for Bochner Riesz summability. However, as we pointed out before, (3.18) can only hold for a much smaller range of exponents. For instance, when $n=2$ the bounds can only hold for at most $1 \leq p \leq 8 / 7$, instead of $1 \leq p \leq 6 / 5$, if any boundary points is geodesically convex. 
To prove Proposition 3.5, we shall require the following straightforward consequences of its hypotheses.

Lemma 3.6. Suppose that (3.18) holds. Suppose also that $\rho \in C(\mathbb{R})$ satisfies $|\rho(\tau)| \leq C_{N}(1+|\tau|)^{-N}$ for some $N>\frac{n}{p}-\frac{n+1}{2}+1$. Assume also that $1 \leq 2^{k} \leq \lambda$. Then there is a uniform constant $C$ so that

$$
\left\|\rho\left(2^{-k}(\lambda-\mathcal{P})\right) f\right\|_{2}+\left\|\rho\left(2^{-k}(\lambda+\mathcal{P})\right) f\right\|_{2} \leq C 2^{k / 2} \lambda^{\frac{n}{p}-\frac{n+1}{2}}\|f\|_{p}, \quad \lambda \geq 1,
$$

where the constant only depends on $C_{N}$ and the constant in (3.18).

Here we are of course using the notation that $\rho(\mathcal{P}) f=\sum_{j} \rho\left(\lambda_{j}\right) e_{j}(f)$.

Proof of Lemma 3.6. If we just use orthogonality, (3.18), and our assumptions on $\rho$ we find that

$$
\begin{aligned}
& \left\|\rho\left(2^{-k}(\lambda-\mathcal{P})\right) f\right\|_{2}^{2}+\left\|\rho\left(2^{-k}(\lambda+\mathcal{P})\right) f\right\|_{2}^{2} \\
& \quad \leq C \sum_{j=0}^{\infty}\left(\sup _{\lambda_{l} \in[j, j+1]}\left|\rho\left(2^{-k}\left(\lambda-\lambda_{l}\right)\right)\right|^{2}+\sup _{\lambda_{l} \in[j, j+1]}\left|\rho\left(2^{-k}\left(\lambda+\lambda_{l}\right)\right)\right|^{2}\right)\left\|\chi_{j} f\right\|_{2}^{2} \\
& \quad \leq C \sum_{j=0}^{\infty}\left(\left(1+2^{-k}|\lambda-j|\right)^{-N}+\left(1+2^{-k}|\lambda+j|\right)^{-N}\right)(1+j)^{2\left(\frac{n}{p}-\frac{n+1}{2}\right)}\|f\|_{p}^{2} .
\end{aligned}
$$

The first term in the right dominates the second term. Since $N>\frac{n}{p}-\frac{n+1}{2}+1$ and $2^{-k} \lambda \geq 1$, by comparing the sums to the corresponding integrals, one sees that both terms on the right can be dominated by the square of the right hand side of (3.20), which finishes the proof.

We now have the main tools needed to prove Proposition 3.5. To be able to rewrite the operators $S_{\lambda}^{\delta}$ in a way that will allow us to use the above estimates we need to relate the operator to the wave equation. For this purpose, we need to compute the Fourier transform of the symbol $\tau \rightarrow\left(1-\tau^{2} / \lambda^{2}\right)_{+}^{\delta}$ of the Bochner Riesz means. We shall use the Bessel function formula,

$$
\int_{-1}^{1} e^{i \tau t}\left(1-\tau^{2}\right)^{\delta} d \tau=\sqrt{\pi} \Gamma(1+\delta)\left(\frac{t}{2}\right)^{-\delta-\frac{1}{2}} J_{\delta+\frac{1}{2}}(t), \quad t>0 .
$$

Recall that as $r \rightarrow \infty$, we have the following asymptotics for Bessel functions of order $m$

$$
J_{m}(r)=\sum_{ \pm} \alpha_{m}^{ \pm}(r) e^{ \pm i r}
$$

where

$$
\left|\partial^{j} \alpha_{m}^{ \pm}(r)\right| \leq C_{j} r^{-j-1 / 2}, \quad r \geq 1, j=0,1,2, \ldots
$$


Therefore, since this Fourier transform is an even function, we can write

$$
\begin{aligned}
S_{\lambda}^{\delta} f & =\sum_{\lambda_{j} \leq \lambda}\left(1-\lambda_{j}^{2} / \lambda^{2}\right)^{\delta} e_{j}(f) \\
& =\frac{1}{2 \pi} \int_{-\infty}^{\infty} \lambda \sqrt{\pi} \Gamma(1+\delta)\left(\frac{\lambda|t|}{2}\right)^{-\delta-\frac{1}{2}} J_{\delta+\frac{1}{2}}(\lambda|t|) \sum_{j} \cos t \lambda_{j} e_{j}(f) d t \\
& =\frac{1}{2 \pi} \int_{-\infty}^{\infty} \sum_{ \pm} \lambda m_{\delta}^{ \pm}(\lambda t) e^{ \pm i \lambda t} \cos t \mathcal{P} f d t
\end{aligned}
$$

where, for every $j,\left|(1+|t|)^{j} \partial_{t}^{j} m_{\delta}^{ \pm}(t)\right| \leq C_{\delta}(1+|t|)^{-1-\delta}$, and hence

$$
\lambda\left|(1+|t|)^{j} \partial_{t}^{j} m_{\delta}^{ \pm}(\lambda t)\right| \leq\left\{\begin{array}{l}
C_{\delta} \lambda, \quad|t| \leq \lambda^{-1} \\
C_{\delta} \lambda^{-\delta}|t|^{-1-\delta}, \quad|t| \geq \lambda^{-1} .
\end{array}\right.
$$

Here, $\mathcal{P}=\sqrt{-\Delta}$, and

$$
u(t, x)=\cos t \mathcal{P} f(x)=\sum_{j=1}^{\infty} \cos t \lambda_{j} e_{j}(f)(x),
$$

is the cosine transform of $f$. Thus, it is the solution of the wave equation

$$
\left(\partial_{t}^{2}-\Delta_{g}\right) u=0, \quad u(0, \cdot)=f, \partial_{t} u(0, \cdot)=0 .
$$

We shall use the finite propagation speed for solutions to the wave equation. Specifically, if $f$ is supported in a geodesic ball $B\left(x_{0}, R\right)$ centered at $x_{0}$ with radius $R$, then $x \rightarrow \cos t \mathcal{P} f$ vanishes outside of $B\left(x_{0}, 2 R\right)$ if $0 \leq t \leq R$.

We shall now proceed to break up the operators $S_{\lambda}^{\delta}$ into a sum of pieces that we can estimate using a combination of (3.20) and Hölder's inequality, along with a "remainder term". This is related to an argument of Fefferman [3] for the Euclidean case, and also an argument of the author [16] for the case of Riemannian manifolds without boundary. The latter argument also relied on the small time parametrix for the wave equation, which is impossible to use in this setting. Instead we use a simpler argument that only uses the finite propagation speed of the wave equation.

Let us first deal with the remainder term. We fix an even function $b \in C^{\infty}(\mathbb{R})$ satisfying $b(t)=0,|t|<1$ and $b(t)=1,|t|>2$, and then set

$$
R_{\lambda}^{\delta} f=\frac{1}{2 \pi} \int_{-\infty}^{\infty} \sum_{ \pm} \lambda m_{\delta}^{ \pm}(\lambda t) b(t) e^{ \pm i \lambda t} \cos t \mathcal{P} f d t .
$$

If $\rho_{\lambda}$ denotes the inverse Fourier transform $t \rightarrow \frac{1}{2} m_{\delta}^{ \pm}(\lambda t) b(t)$, then

$$
R_{\lambda}^{\delta} f=\rho_{\lambda}(\lambda-\mathcal{P}) f+\rho_{\lambda}(\lambda+\mathcal{P}) f .
$$

Using (3.22), one finds that for fixed $\delta$ one has the uniform bounds

$$
\left|\rho_{\lambda}(\tau)\right| \leq C_{N} \lambda^{-\delta}(1+|\tau|)^{-N}
$$


for every $N$. Hence, (3.20) and Hölder's inequality imply that for every $\lambda \geq 1$

$$
\left\|R_{\lambda}^{\delta} f\right\|_{p} \leq C\left\|R_{\lambda}^{\delta} f\right\|_{2} \leq C \lambda^{\frac{n}{p}-\frac{n+1}{2}-\delta}\|f\|_{p}
$$

which shows that the remainder terms $R_{\lambda}^{\delta}$ are uniformly bounded when $\delta \geq$ $\frac{n}{p}-\frac{n+1}{2}$.

If we let $a(t)=1-b(t)$, so that $a(t)=1$ for $|t|<1$ and 0 for $|t|>2$, we would be done if we could prove the same for

$$
\tilde{S}_{\lambda}^{\delta} f=\frac{1}{2 \pi} \int_{-\infty}^{\infty} \sum_{ \pm} \lambda m_{\delta}^{ \pm}(\lambda t) a(t) e^{ \pm i \lambda t} \cos t \mathcal{P} f d t .
$$

To do this, we shall make a dyadic decomposition of the integral. Fix $\beta \in C_{0}^{\infty}(\mathbb{R})$ satisfying $\beta(t)=0 t \notin[1 / 2,4]$ and $\sum_{-\infty}^{\infty} \beta\left(2^{-j} t\right)=1, t>0$. We then set $\beta_{0}(t)=\sum_{j=0}^{\infty} \beta\left(2^{-j}|t|\right)$ so that $\beta_{0}$ is smooth and satisfies $\beta_{0}(t)=0,|t|>2$. We then define for $j=1,2, \ldots$

$$
\tilde{S}_{\lambda, j}^{\delta} f=\frac{1}{2 \pi} \int_{-\infty}^{\infty} \sum_{ \pm} \lambda m_{\delta}^{ \pm}(\lambda t) \beta\left(\lambda 2^{-j}|t|\right) a(t) e^{ \pm i \lambda t} \cos t \mathcal{P} f d t
$$

and

$$
\tilde{S}_{\lambda, 0}^{\delta} f=\frac{1}{2 \pi} \int_{-\infty}^{\infty} \sum_{ \pm} \lambda m_{\delta}^{ \pm}(\lambda t) \beta_{0}(\lambda t) a(t) e^{ \pm i \lambda t} \cos t \mathcal{P} f d t,
$$

so that $\tilde{S}_{\lambda}^{\delta} f=\sum_{j \geq 0} \tilde{S}_{\lambda, j}^{\delta} f$. Note that, because of the support properties of $a(t)$, $\tilde{S}_{\lambda, j}^{\delta} f$ vanishes if $j$ is larger than a fixed multiple of $\log \lambda$.

We claim that if $\delta>\frac{n}{p}-\frac{n+1}{2}$ is fixed then

$$
\left\|\tilde{S}_{\lambda, j}^{\delta} f\right\|_{p} \leq C 2^{-\left(\delta-\left(\frac{n}{p}-\frac{n+1}{2}\right)\right) j}\|f\|_{p}
$$

where $C$ is independent of $\lambda$ and $j$. This would of course complete the missing step of obtaining the uniform boundedness of the $\tilde{S}_{\lambda}^{\delta}$ for $\delta>\frac{n}{p}-\frac{n+1}{2}$.

To prove this estimate we shall exploit the fact that the finite propagation speed of the wave equation mentioned before implies that the kernels of the operators, $\tilde{S}_{\lambda, j}^{\delta}(x, y)$ must satisfy

$$
\tilde{S}_{\lambda, j}^{\delta}(x, y)=0, \quad \text { if } \operatorname{dist}(x, y) \geq 8\left(2^{j} \lambda^{-1}\right),
$$

since $\cos t \mathcal{P}$ will have a kernel that vanishes on this set when $t$ belongs to the support of the integral defining $\tilde{S}_{\lambda, j}^{\delta}$. Because of this, in order to prove (3.23), it suffices to show that for all geodesic balls $B_{R_{\lambda, j}}$ of radius $R_{\lambda, j}=\lambda^{-1} 2^{j}$ one has the bounds

$$
\left\|\tilde{S}_{\lambda, j}^{\delta} f\right\|_{L^{p}\left(B_{R_{\lambda, j}}\right)} \leq C 2^{-\left(\delta-\left(\frac{n}{p}-\frac{n+1}{2}\right)\right) j}\|f\|_{p}
$$

for the $L^{p}$ norms over $B_{R_{\lambda, j}}$, with $C$, as before, being independent of $\lambda$ and $j$. However, by Hölder's inequality,

$$
\left\|\tilde{S}_{\lambda, j}^{\delta} f\right\|_{L^{p}\left(B_{R_{\lambda, j}}\right)} \leq C\left(\lambda^{-1} 2^{j}\right)^{\frac{n}{p}-\frac{n}{2}}\left\|\tilde{S}_{\lambda, j}^{\delta} f\right\|_{L^{2}(M)},
$$


and so we would be done if we could show that

$$
\left\|\tilde{S}_{\lambda, j}^{\delta} f\right\|_{2} \leq C\left(\lambda^{-1} 2^{j}\right)^{-\frac{n}{p}+\frac{n}{2}} 2^{-\left(\delta-\left(\frac{n}{p}-\frac{n+1}{2}\right)\right) j}\|f\|_{p}=C \lambda^{\frac{n}{p}-\frac{n+1}{2}}\left(\lambda 2^{-j}\right)^{\frac{1}{2}} 2^{-\delta j}\|f\|_{p} .
$$

To prove this for $j=1,2, \ldots$ we note that, by (3.22), the inverse Fourier transform of $t \rightarrow \frac{1}{2} \lambda m_{\delta}(\lambda t) \beta\left(\lambda 2^{-j}|t|\right)$ behaves like that of $\lambda^{-\delta}|t|^{-1-\delta} \beta\left(\lambda 2^{-j}|t|\right)$. Since the dyadic cutoff localizes to $|t| \approx \lambda^{-1} 2^{j}$, we conclude that we can write

$$
\tilde{S}_{\lambda, j}^{\delta} f=2^{-j \delta} \rho_{\lambda, j}\left(\lambda^{-1} 2^{j}(\lambda-\mathcal{P})\right) f+2^{-j \delta} \rho_{\lambda, j}\left(\lambda^{-1} 2^{j}(\lambda+\mathcal{P})\right) f
$$

where the $\rho_{\lambda, j}$ satisfy the uniform bounds

$$
\left|\rho_{\lambda, j}(\tau)\right| \leq C_{N}(1+|\tau|)^{-N}
$$

for every $N$. Because of this, the estimates (3.25) with $j=1,2, \ldots$ just follow from (3.20) with $2^{k}$ being replaced by $\lambda 2^{-j}$. Since the estimate for $j=0$ follows from the same argument, the proof is complete.

\section{References}

[1] V.G. Avakumovic, Über die Eigenfunktionen der Schwingungsgleichung, Acad. Serbe Sci. Publ. Inst. Math. 4 (1952), 95-96.

[2] _ Über die Eigenfunktionen auf geschlossenen Riemannschen Mannigfaltigkeiten, Math. Z. 65 (1956), 327-344.

[3] C. Fefferman, Inequalities for strongly singular convolution operators, Acta Math. 124 (1970), 9-36.

[4] D. Grieser, $L^{p}$ bounds for eigenfunctions and spectral projections of the Laplacian near concave boundaries, PhD thesis, UCLA, 1992.

[5] _ Uniform bounds for eigenfunctions of the Laplacian on manifolds with boundary, to appear in Comm. PDE.

[6] M.H. Protter and H.F. Weinberger, Maximum principles in differential equations, Prentice-Hall, Inc., Englewood Cliffs, NJ, 1967.

[7] L. Hörmander, The spectral function of an elliptic operator, Acta Math. 121 (1968), 193-218.

[8] _ The analysis of linear partial differential operators. III. Pseudodifferential operators, Grundlehren der Mathematischen Wissenschaften, 274. Springer-Verlag, Berlin, 1985.

[9] _ On the Riesz means of spectral functions and eigenfunction expansions for elliptic differential operators, Some recent advances in the basic sciences, Yeshiva Univ., New York, (1965-1966), 155-202.

[10] V. J. Ivrii, Microlocal analysis and precise spectral asymptotics, Springer Monographs in Mathematics. Springer-Verlag, Berlin, 1998.

[11] B. M. Levitan, Über die Entwicklung nach den Eigenfunktionen des Laplaceschen Operators, Dokl. Akad. Nauk SSSR 90 (1953), 133-135.

[12] Pham The Lai, Meilleurs estimations asymptotiques des restes de la fonction spectrale et des valeurs propres relatifs au laplacien, Math. Scand. 48 (1981), 5-31.

[13] R. T. Seeley, An estimate near the boundary for the spectral function of the Laplace operator, Amer. J. Math. 102 (1980), no. 5, 869-902.

[14] H.F. Smith and C. D. Sogge, On the critical semilinear wave equation outside of convex obstacles, J. Amer. Math. Soc. 8 (1995), no. 4, 879-916.

[15] C. D. Sogge, Concerning the $L^{p}$ norm of spectral clusters for second-order elliptic operators on compact manifolds, J. Funct. Anal. 77 (1988), no. 1, 123-134. 
[16] On the convergence of Riesz means on compact manifolds, Ann. of Math. (2) 126 (1987), no. 2, 439-447.

[17] $\ldots$ Fourier integrals in classical analysis, Cambridge Tracts in Mathematics, 105. Cambridge University Press, Cambridge, 1993.

[18] C. D. Sogge and S. Zelditch, Riemannian manifolds with maximal eigenfunction growth, to appear in Duke Math. J.

Department of Mathematics, The Johns Hopkins University, Baltimore, MD 21218, U.S.A.

E-mail address: sogge@jhu.edu 